\title{
GEM-based detectors for direct detection of low-mass WIMP, solar axions and narrow resonances (quarks).
}

\author{
B.M.Ovchinnikov, V.V.Parusov* \\ Institute of Nuclear Research of RAS, Moscow, Russia. \\ *Corresponding author: parusov@inr.ru
}

\begin{abstract}
.
Gas electron multipliers (GEMs) with wire (WGEMs) or metal electrodes (MGEMs), which don't use any plastic insulators between electrodes are created. The chambers containing MGEMs (WGEMs) with pin-anodes are proposed as detectors for searching of spin-dependent interactions between Dark Matter (DM) particles and gases with nonzero-spin nuclei $\left(\mathrm{H}_{2}, \mathrm{D}_{2},{ }^{3} \mathrm{He},{ }^{21} \mathrm{Ne}, \mathrm{CF}_{4}, \mathrm{CH}_{4}\right.$, etc.). In this paper, we present a review of such chambers.

For investigation of the gas mixtures $\mathrm{Ne}+10 \% \mathrm{H}_{2}, \mathrm{H}_{2}\left(\mathrm{D}_{2}\right)+3 p p m T M A E$, the chamber containing WGEM with pin-anode detection system was constructed. In this paper we present the results of an experimental study of these gaseous mixtures exited by an $\alpha$ - source. Mixture of $\mathrm{Ar}+40 \mathrm{ppm} \mathrm{C}_{2} \mathrm{H}_{4}$ and mixture $50 \% \mathrm{Xe}+50 \% \mathrm{CF}_{4}$ have been investigated. The spatial distributions of photoelectron clouds produced by primary scintillations on $\alpha$ - and $\beta$-particle tracks, as well as the distributions of photoelectron clouds due to photons from avalanches at the pin-anode, have been measured for the first time.
\end{abstract}

In our experiments as another filling of the chambers for search of low-mas WIMP $\left(<10 \mathrm{GeV} / \mathrm{c}^{2}\right)$, solar neutrino and solar axions with spin-dependent interaction we propose to use the mixtures: $D_{2}+$ 3ppmTMAE, ${ }^{3} \mathrm{He}+3 \% \mathrm{CH}_{4},{ }^{21} \mathrm{Ne}+10 \% \mathrm{H}_{2}$, at pressure 10-17 bar. And in our experiment with liquid gases is used the mixtures with ${ }^{19} \mathrm{~F}\left(\mathrm{LAr}+\mathrm{CF}_{4}, \mathrm{LXe}+\mathrm{CF}_{4}\right)$ and mixture $\mathrm{LCH}_{4}+40 \mathrm{ppm}$ TMAE.

The time projection chamber (TPC) with the mixture $D_{2}+3 p p m T M A E$ filling allow to search of spin-dependent interactions of solar axions and deuterium. As well as we present the detecting systems for search of narrow pp-resonances (quarks) in accelerators experiments.

Keywords: MGEM, pin-anodes, Low-Mass WIMP, Axions, Quarks, TPC, SD-interactions, $\mathrm{H}_{2}, \mathrm{CF}_{4}$, $\mathrm{CH}_{4}$, TMAE.

\section{Introduction}

More than forty years ago G. Charpak and F. Sauli have introduced their Multi-Step Chambers to overcome limitations of gain in Parallel-Plate and Multi-Wire Proportional Chambers (MWPC) [1, 2]. Currently there are different types of detectors for fast detection and localization of charged particles exist. One of them is a Gas Electron Multiplier (GEM) [2-4]. More coarse macro-patterned detectors are thick-GEMs (THGEM) [5-7] or patterned resistive thick GEM devices (RETGEM) [8].

However, the most essential disadvantage of GEMs consists in their low reliability and stability. The matter is that in a process of dispersion of the GEM's cathode electrodes by positive ions of proportional avalanches in GEM with metal or high-resistive electrodes (RETGEM), a sedimentation of the sprayed carrying-out material on the walls of holes with subsequent leaks and breakdowns between 
electrodes takes place. Micro-pattern gaseous detectors (MPGD), due to their tiny electrode structure and small avalanche gaps, are very fragile and can be easily damaged by sparks appearing at high operational gains (typically at gains of $10^{4}$ or slightly more) [7].

Therefore, we were concentrated on development of more robust designs of GEM detectors with wire (WGEM) [9-12] or metal electrodes (MGEM) [13, 14]. In our next works [15-18, 21, 25, 37] it was suggested that the search for spin-dependent WIMP-nucleon interactions with help of detecting system GEM + pin-anodes can be performed.

\section{Gas electron multipliers with metal electrodes.}

The idea of GEM without plastic insulators was first mentioned in our works [9-13]. In the paper [14] we have described a novel concept of MGEMs with etching holes. In Fig.1 we present the designs of metal GEMs.

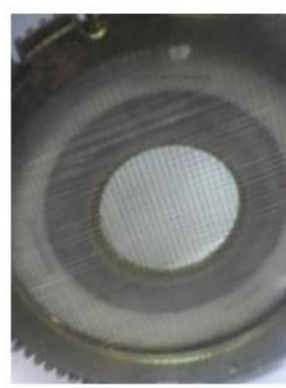

a)

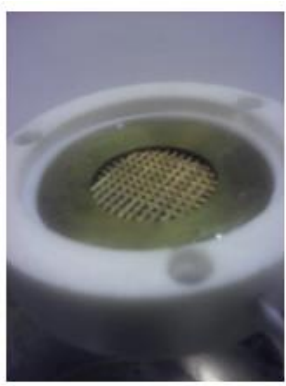

b)

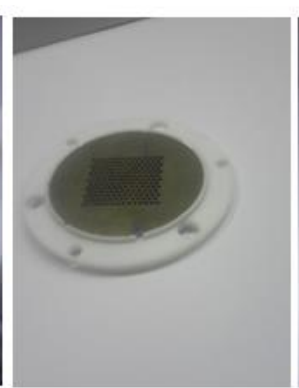

c)

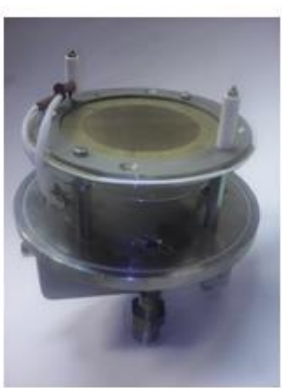

d)

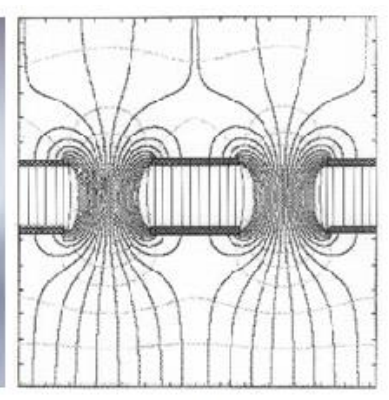

e)
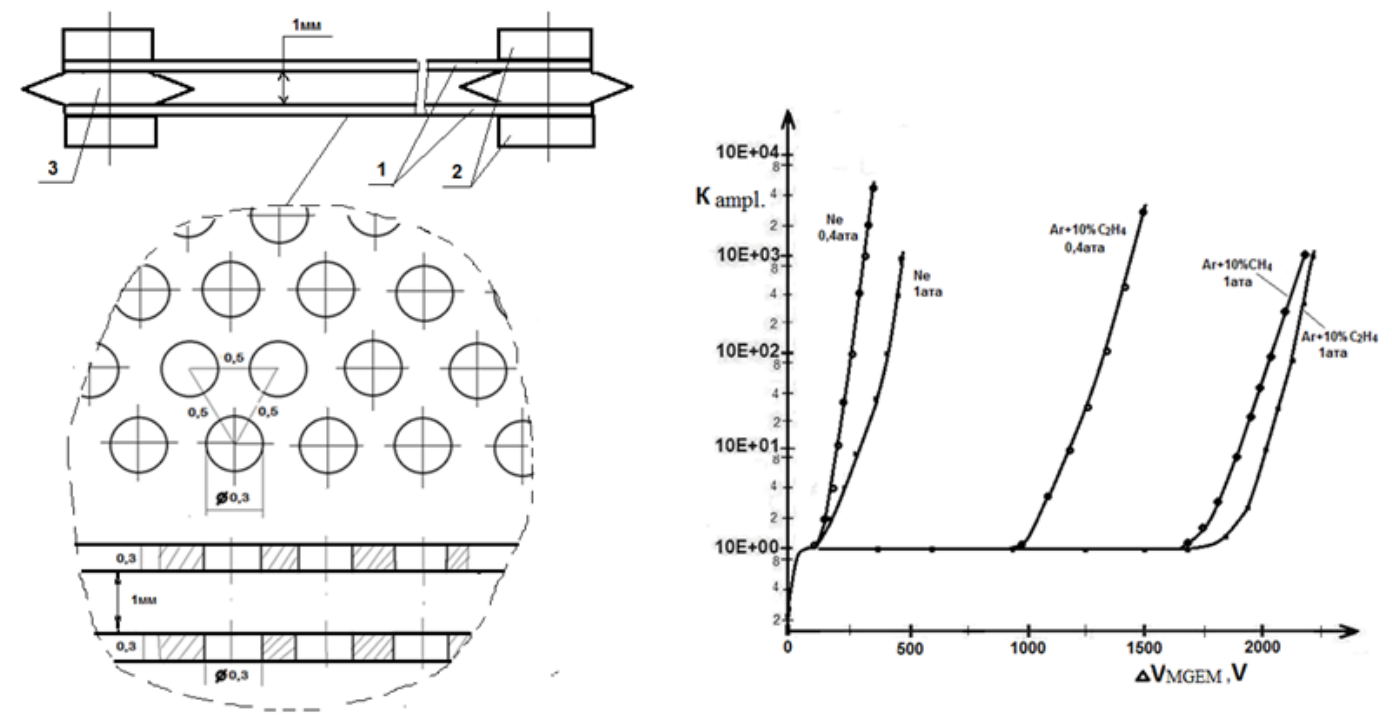

Fig.1. Designs of metal GEMs [9-13]: a,b) GEMs with wire electrodes; c) GEM with metal electrodes; d) GEM with the etching of holes and result of test [14]; e) electric field structure in the GEM. 


\section{Chamber with system WGEM + pin-anodes.}

In Fig. 2 we present the wire gas electron multiplier (WGEM) in combination with pin-anode and results of test (Fig.3). In this section we discuss the operation principle of MWPC, GEM and system GEM + pin-anode (Fig.4).
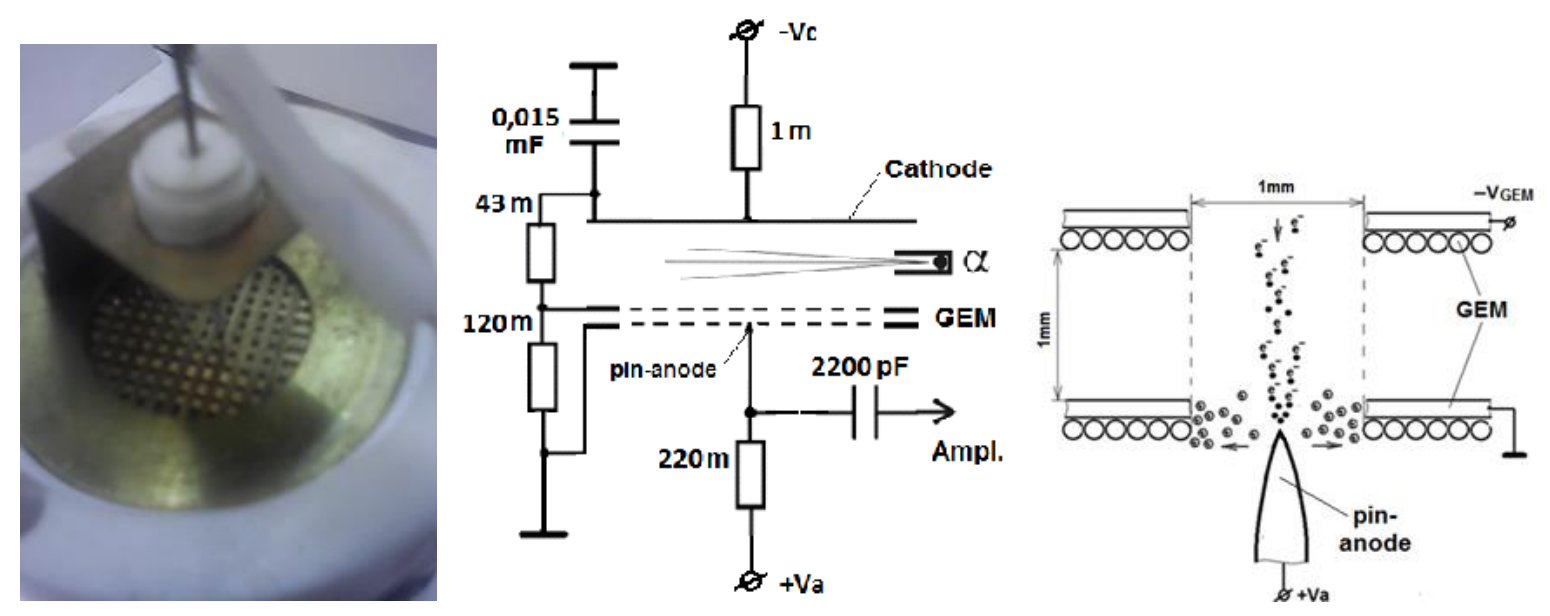

Fig.2. Detection system for testing of WGEM + pin-anode with diagram of the travel of positive ions from avalanches developed at the pin and electrons being collected at the pin $[16,17,21,25]$.
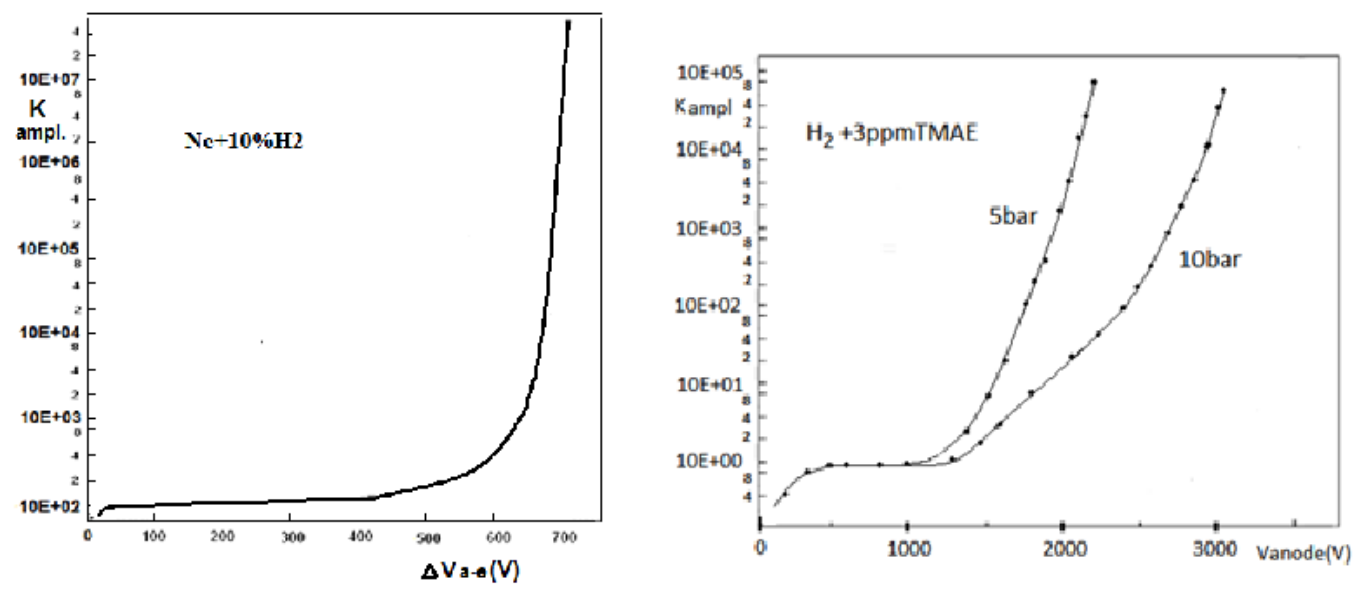

Fig.3. Measured amplification factor of a system WGEM + pin-anode with mixtures: $\mathrm{Ne}+10 \% \mathrm{H}_{2}$ (1 bar) [21] and $\mathrm{H}_{2}+3$ ppm TMAE $(5,10$ bar) [25].

The operation principle of Multi-Wire Proportional Chambers (a), GEM (b) and system GEM + pin-anode (c) is illustrated in Fig.4. The factors which have allowed us to obtain high electron multiplication factors in the GEM + pin-anode system are as follows:

(1) High electric field strength in the system GEM + pin-anode makes it possible to obtain a big length of electron avalanche and high value of the electron multiplication factor $\left(10^{6}-10^{7}\right)$; 
(2) Positive ions from the avalanche at the pin are transferred by the electric field, mainly, to the walls of the hole in which the pin is located and, in smaller quantities, towards the ionization electrons being collected at the pin, which rules out the possibility of streamers being developed at the interface

(3) For GEM (see technology b) extraction efficiency decrease at low transfer fields values due to a worst electron extraction capability from the lower side of the GEM [32];

(4) Absence of a plastic insulation excludes the emergence of leakage current and spark breakdown between electrodes. Accidental spark events in such system don't lead to their failure as positive ions quickly move away from breakdown by a strong electric gap field.

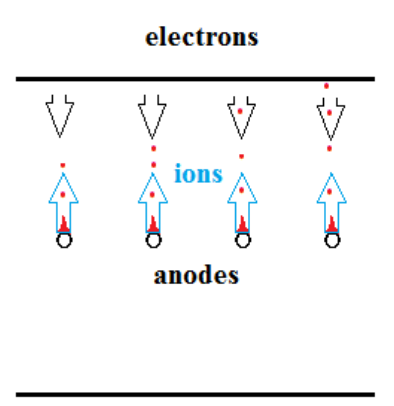

a) MWPC

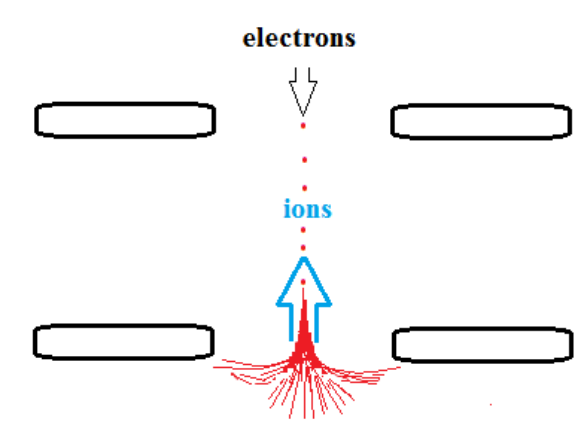

b) GEM

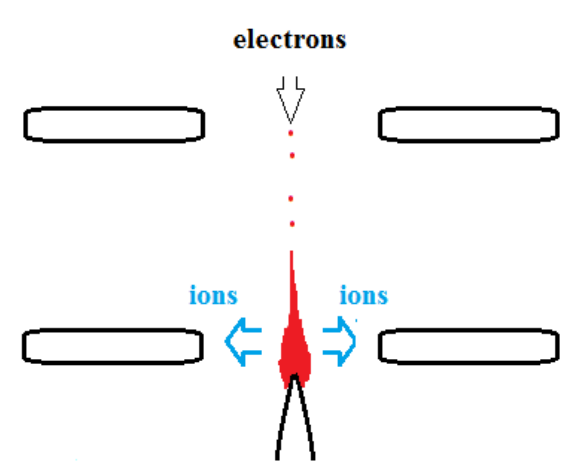

c) GEM+pin-anode

Fig.4. Operation principle of Multi-Wire Proportional Chambers (a), GEM (b) and GEM+pinanode (c). Electron avalanches are shown for three technologies $(a, b, c)$; red paths are electron trajectories, also the drift of ions is indicated (blue paths).

\section{A short review of chambers with system GEM + pin-anodes for direct detection of WIMP.}

In work [15] the idea of focusing screen with holes + system pin-anodes already was shown. The wire gas electron multipliers in combination with pin-anodes are proposed for detection of events:

(1) In the gas phase of a double-phase argon (xenon) chamber [16, 17]. Hydrogen with a concentration of $10 \%$ is added to argon to eliminate feedbacks via photons emitted by excited argon molecules in avalanche development processes during detection of events in the gaseous argon.

(2) In chamber gas mixture $\mathrm{Ne}+10 \% \mathrm{H}_{2}$ for direct detection of WIMP with mass $\leq 0.5$ $\mathrm{GeV} / \mathrm{c}^{2}[21]$. Based on the work [22], where in a spherical proportional detector the energy threshold is about $100 \mathrm{eV}$, while the amplification factor of the detecting system is about $10^{4}$, we estimate the threshold of our experiment to be about 100 $\mathrm{eV} \cdot 10^{4} / 5 \cdot 10^{7}<1 \mathrm{eV}$. As another filling of the chamber is used the mixture ${ }^{21} \mathrm{Ne}+10 \% \mathrm{H}_{2}$ for search of spin-dependent interaction [23, 35, 36].

(3) In chamber with gas mixture $\mathrm{H}_{2}+3 p p m$ TMAE for direct detection of WIMP with mass $\leqslant 10 \mathrm{GeV} / \mathrm{c}^{2}$ and solar axions [25]. As another filling of the chamber is used the mixture deuterium $\left(D_{2}\right)+3 p p m$ TMAE. Because the energy of axion is equal to $\sim 1 \mathrm{keV}$ [26], is transfers the energy to recoil deuterium. The $\mathrm{H}_{2}\left(\mathrm{D}_{2}\right)$-filling provides the electron background suppression, because the recoil protons in $\mathrm{H}_{2}$-medium have the short track [30], as distinguished from background electrons. 
(4) In double-phase chambers with $\mathrm{LXe}+\mathrm{CF}_{4}$ and $\mathrm{LCH}_{4}+\mathrm{TMAE}$ filling (section 7). In our experiments [15-18] with liquid gases mixtures is used the mixtures for search of spindependent WIMP-nucleon interacting.

(5) In scintillation and ionization fast chambers with mixture $\mathrm{Xe}+\mathrm{CF}_{4}$ for accelerators experiments (section 9).

In the time projection chamber (TPC) with the mixture $\mathrm{D}_{2}+3 p p m T M A E$ filling we use of twostep system GEM + MWPC (section 8).

\section{A method for background reduction in experiments for direct detection of WIMPs.}

To suppress the $\beta, \gamma$ and n0 backgrounds, we proposed [18] a addition in liquid argon of photosensitive dopants and a comparison of scintillation (S1) and ionization signals (S2) for every event is suggested. The addition in liquid Ar of photosensitive TMA, TMG or $\mathrm{C}_{2} \mathrm{H}_{4}$ [19] and suppression of triplet component of scintillation signals ensures the detection of scintillation signals with high efficiency and provides a complete suppression of the electron background.

In work [20] we investigated of scintillation (S1) and ionization signals (S2) on a mixture of Ar + 40 ppm $\mathrm{C}_{2} \mathrm{H}_{4}$ at a pressure of 5 bar.

The measurements were taken inside the chamber similar a chamber with pin-anode (see Fig. 5 left). The mixture was irradiated with $\alpha(239 \mathrm{Pu})$ and $\beta(63 \mathrm{Ni})$ particles. The chamber was used with potentials of pin-anode $\mathrm{Va}=1300 \mathrm{~V}, \mathrm{Kamp} \sim 10^{4}(\beta)$ and $\mathrm{Va}=520 \mathrm{~V}, \mathrm{Kamp}=30(\alpha)$.

Peak S1 (Fig. 5 right) is associated with the cloud of photoelectrons from the chamber volume due to scintillation photons with $\lambda=128 \mathrm{~nm}$, which are emitted upon excitation of argon atoms with $\alpha(\beta)$ particles: hv $+\mathrm{C}_{2} \mathrm{H}_{4} \rightarrow \mathrm{C}_{2} \mathrm{H}_{4}{ }^{*}+\mathrm{e}-$. Peak S2 is due to ionization electrons from $\alpha(\beta)$ particles. Peak S2 fluctuates in the amplitude for different events, since the $\beta$-particle spectrum lies in the range of $E^{\mathrm{min}}$ $E^{\max }(17-67 \mathrm{KeV})$. Peak S3 can be attributed to the cloud of photoelectrons from the chamber volume produced by photons from avalanches at the pin-anode.

The results obtained in our study suggest that it is possible to develop large volume detectors capable of detecting scintillations with a $100 \%$ geometrical efficiency, by contrast to the well known detection techniques based on photomultipliers having efficiency of only a few percent [27].
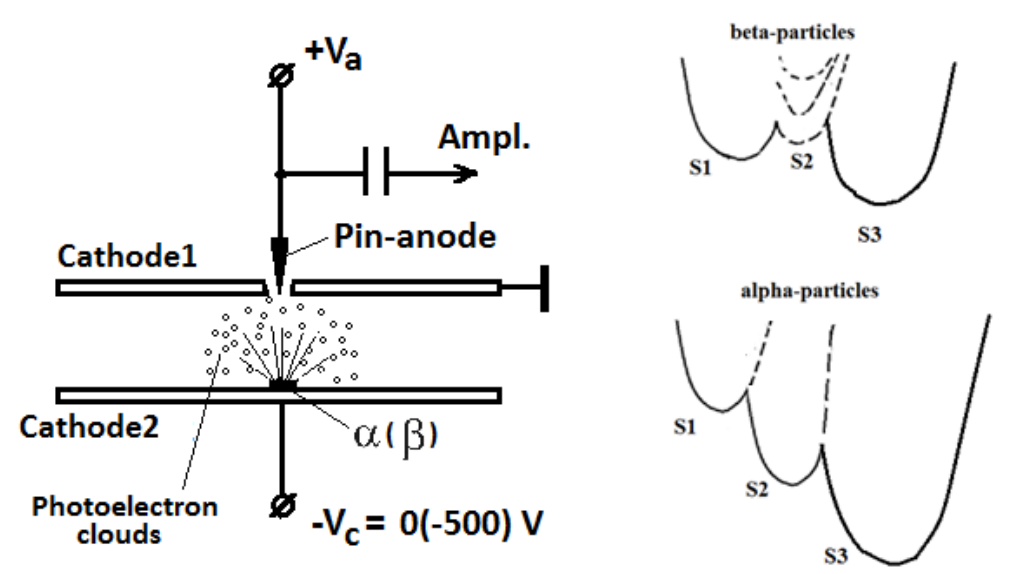

Fig. 5. The chamber filled with the mixture of $\mathrm{Ar}+40 \mathrm{ppm} \mathrm{C}_{2} \mathrm{H}_{4}$ at a pressure of 5 bar. The mixture was irradiated with $\alpha(239 \mathrm{Pu})$ and $\beta$ (63Ni) particles. The chamber was used in potentials of pinanode $\mathrm{Va}=1300 \mathrm{~V}$, Kamp $\sim 10^{4}(\beta$-particles) and Va $=520 \mathrm{~V}, \mathrm{Kamp}=30$ ( $\alpha$-particles). $-\mathrm{Vc}=0 \mathrm{~V}$ 


\section{Chambers with $\mathrm{Xe}+\mathrm{CF}_{4}(1: 1)$ gas mixture.}

\subsection{The ratio S1/S2 for $\beta$ and $\alpha$-particles. Nanosecond timing scintillation chamber with mixture $\mathrm{Xe}+\mathrm{CF}_{4}$ filling.}

Recently we have investigated a scintillation signal (S1) and a ratio scintillation to ionization signal (S1/S2) for $\beta$ and $\alpha$-particles on prototype of fast chamber (Fig.6) with mixture Xe+CF $(1: 1)$ filling at a pressure of 10 bar. This chamber was irradiated with $\alpha(239 \mathrm{Pu})$ and $\beta$ (63Ni) particles. The scintillations signals (S1) were measured separately of photomultiplier (PMT-85) with fast shifter (OB205). The ionizations signals (S2) were measured on anode of chamber. The addition in $\mathrm{Xe}$ of $\mathrm{CF}_{4}$ and suppression of long triplet component of signals ( $27 \mathrm{~ns})$ ensures the detection of scintillation signals with high speed (1ns).

A shifter OB-205 has a maximum sensitivity range of $185 \mathrm{~nm}$ and converts with high efficiency of UV-light in visible light (420 nm). And also he have fast luminescence lifetime ( 1ns) and high photoluminescence quantum yield (99\%). The measurements S1 signal used a fast amplifier and an oscilloscope Le Croy-232. Electronegative impurities $\mathrm{O}_{2}, \mathrm{C}_{2} \mathrm{~F}_{4}$ and $\mathrm{C}_{3} \mathrm{~F}_{8}$ were removed from the gases an a purification system to a level of $10^{-8} \mathrm{O}_{2}$ equivalent $(0,01 \mathrm{ppm})$. The entire system (chamber+ gas system) was checked by the "ISTOK" gas analyzer [32] for the presence of known electronegative impurities, which were not detected as a result.

For alpha particles, the ratio $\mathrm{A}=\mathrm{S} 1 / \mathrm{S} 2$ was 0.63 and $\mathrm{B}=\mathrm{S} 1 / \mathrm{S} 2$ for beta particles 25 . The ratio beta to alpha was $B / A=25 / 0,63=40$.

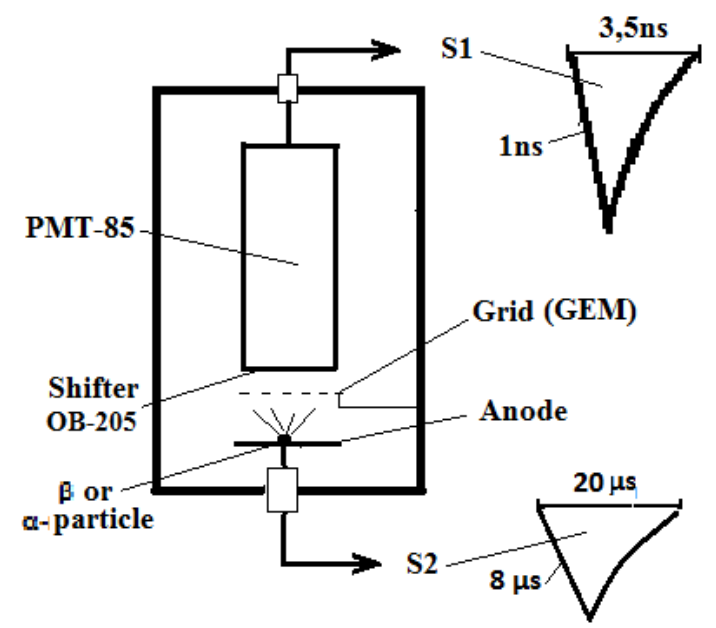

Table 2.

\begin{tabular}{|c|c|c|c|c|c|l|}
\hline $\mathrm{U}(\mathrm{V})$ & 1000 & 1100 & 1200 & 1300 & 1400 & $\mathrm{~T}(\mathrm{~ns})$ \\
\hline $\mathrm{Xe}+\mathrm{CF} 4$ & 200 & 500 & 900 & 1300 & 1800 & lns \\
\hline $\mathrm{CF} 4$ & 56 & 120 & 200 & 400 & 800 & 2ns \\
\hline Ar+CF4 & 15 & 25 & 70 & 120 & 290 & 3ns \\
\hline Ne+CF4 & 20 & 70 & 180 & 275 & 620 & 6ns \\
\hline $\mathrm{Xe}$ & 600 & 1000 & 2200 & 4500 & 7000 & $27 \mathrm{~ns}$ \\
\hline
\end{tabular}

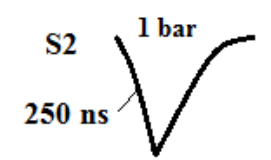

Fig.6. Left: prototype of fast chamber with mixture $\mathrm{Xe}+\mathrm{CF}_{4}$ (1:1) filling. Right: amplitude of scintillations signals ( $\mathrm{mV}$ ), which measured of photomultiplier for $\alpha$-particles on different gases at pressure 10 bar. $U(\mathrm{~V})$ - voltage of PMT-85 (1000V - 1400V). The potential applied to anode is $\mathrm{Va}=$ $+400 \mathrm{~V}$, the gap of anode - grid is $8 \mathrm{~mm}$.

For mixture $A r+C F_{4}(1: 1)$ at a pressure 10 bar, the speed is 3 ns. For mixture $\mathrm{Ne}+\mathrm{CF}_{4}(1: 1)$ at a pressure 10 bar, the speed is $6 \mathrm{~ns}$ (see Table 2). To get a electron multiplication factor $\left(10^{3}\right)$ and large electroluminescence signals, the chamber instead of a grid is set to WGEM [9]. And to get a large electron multiplication factor $\left(10^{4}-10^{5}\right)$ and large ionization anode signals, the chamber instead of a anode is set to system GEM+pin-anodes $[16,17,21,25]$. 


\subsection{The chamber with hole + pin-anode with $\mathrm{Xe}+\mathrm{CF}_{4}$ filling. The measurements photoelectron} signals for $\alpha$ and $\beta$ - particles.

The measurements were taken in the pin-anode chamber (Fig.7 left) [20]. The chamber was used in two operating modes at cathode potentials Vc $=0$ and $-500 \mathrm{~V}$. In both cases, cathode $\mathrm{C} 2$ was grounded. The results obtained thereby are presented in Fig.7 right. The wide peaks observed when $\alpha$ and $\beta$ events were detected at $V c=0$ can be attributed to clouds of photoelectrons due to primary scintillations on particle tracks and to photoelectrons produced by photons from avalanches at the pin anode, as well as to ionization electrons from tracks of $\alpha$ and $\beta$ particles (Fig.8). Since the concentration of photosensitive dopant $\mathrm{CF}_{4}$ was high, all three peaks merged into a single wide peak, by contrast to the spectrum from the mixture of $\mathrm{Ar}+40 \mathrm{ppm} \mathrm{C}_{2} \mathrm{H}_{4}$ in which these three peaks are recorded separately (see section 5.3).

Apparently, $\mathrm{CF}_{4}$ photoionization takes place in the mixture of $\mathrm{Xe}+\mathrm{CF}_{4}: \mathrm{hv}\left(\mathrm{Xe}_{2}{ }^{*}\right) \rightarrow \mathrm{CF}_{4} \rightarrow \mathrm{e}^{+}+\mathrm{F}+$ $\mathrm{CF}_{3}{ }^{-}$. When a negative potential of $-500 \mathrm{~V}$ is applied to chamber cathode $\mathrm{C} 1$, all photoelectrons gather on chamber cathode $\mathrm{C} 2$, and only ionization electrons from $\alpha$ and $\beta$ particles are detected at the pin anode. Figure 7 (right) present the multiplication factor of ionization electron at the pin-anode in the chamber from $\alpha$ and $\beta$ particles tracks as a function of the anode potential in the mixture $\mathrm{Xe}+\mathrm{CF}_{4}(1: 1)$ at pressures of 1 and 10 bar. In these measurements, voltage $V_{c}=-500 \mathrm{~V}$ was applied to cathode $\mathrm{C} 1$, and cathode $\mathrm{C} 2$ was grounded. For $1 \mathrm{bar}$, the maximum electron multiplication factor equal to $3 \times 10^{4}$ was obtained for $\beta$ particles and $K \max =$ was obtained for $\alpha$-particles.

The use of $\mathrm{CF}_{4}$ dopant in noble gas with the aim of increasing the electron drift velocity was described in numerous papers [20]. Our results have demonstrated that $\mathrm{CF}_{4}$ is a photosensitive dopant for $\mathrm{Xe}$. As a result, it is possible to detect scintillations in a chamber filled with a mixture of $\mathrm{Xe}+\mathrm{CF}_{4}$ with a $100 \%$ geometrical efficiency, with is required in the experiment of the search for DM in the Universe for development of detectors with a high mass and complete suppression of background due to $\mathrm{Kr}^{85}$ and external $\boldsymbol{\gamma}$-rays. As well fluorine-19 has a large spin-dependent WIMP-proton cross-section [35, 36, 37].
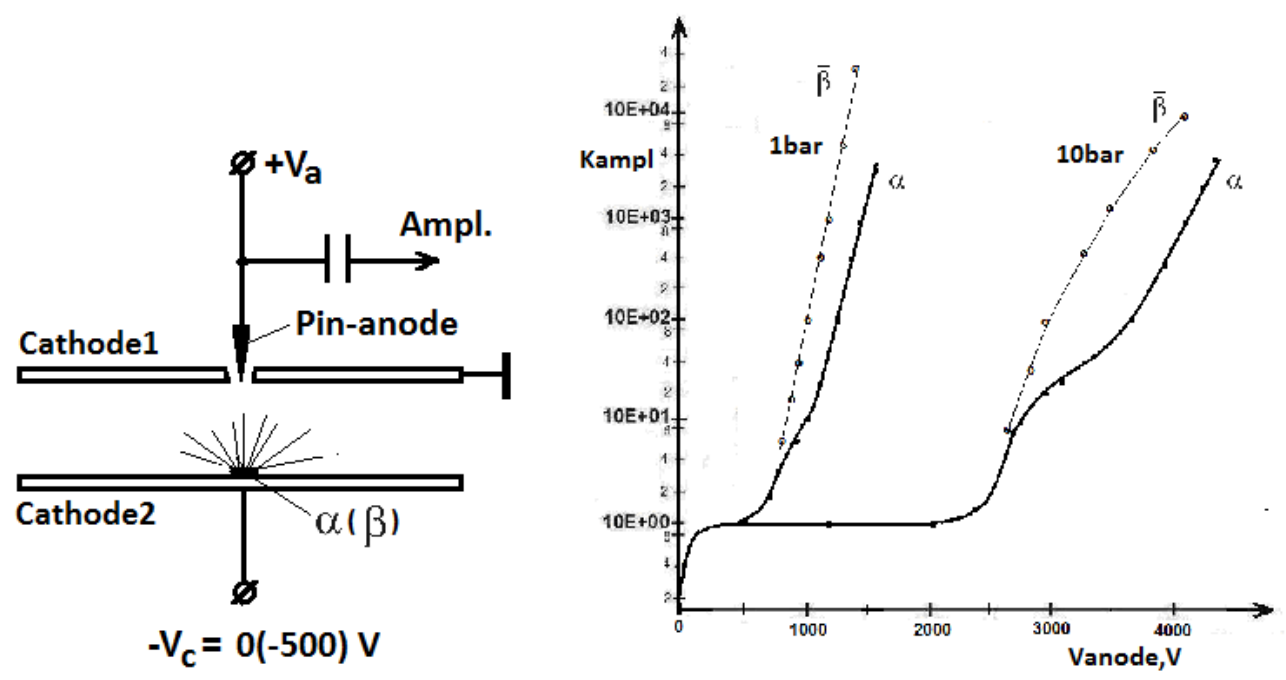

Fig.7 Left: the chamber with $\mathrm{Xe}+\mathrm{CF}_{4}$ (1:1) filling [20]. Right: the multiplication factor of ionization electron at the pin-anode in the chamber from $\alpha(239 \mathrm{Pu})$ and $\beta(63 \mathrm{Ni})$ particles tracks as a function of the anode potential $(0-4500 \mathrm{~V})$. 
a)

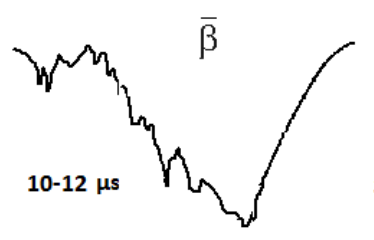

b)

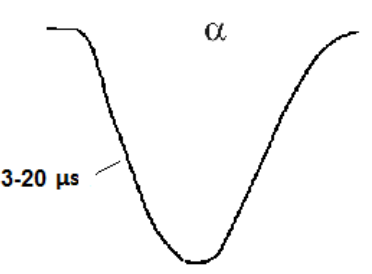

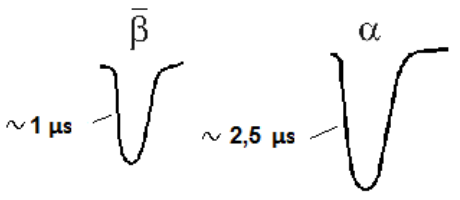

Fig.8. The signals of chamber with the mixture of $\mathrm{Xe}+\mathrm{CF}_{4}$ (1:1) filling at a pressure of 10 bar after irradiation with $\alpha$ and $\beta$ particles at $V a=3000-4000 \mathrm{~V}$ and (a) $\mathrm{Vc}=0 \mathrm{~V}$, (b) $\mathrm{Vc}=-500 \mathrm{~V}$.

\section{Search for spin-dependent WIMP-nucleon interactions.}

\subsection{Double-phase xenon chamber with a system GEM +pin-anodes.}

In particle physics, the lightest supersymmetric particle (LSP) is the generic name given to the lightest of the additional hypothetical particles found in supersymmetric models. In models with R-parity conservation, the LSP is stable; in other words, it cannot decay into any Standard Model particle, since all SM particles have the opposite R-parity. There is extensive observational evidence for an additional component of the matter density in the universe, which goes under the name dark matter. The LSP of supersymmetric models is a dark matter candidate and is a weakly interacting massive particle (WIMP).

From the elementary particle physics in the framework of the standard Bing Bang nucleosynthesis model one infers that DM consists manly of WIMPs: massive neutrinos, axions and particles predicted by SUSY.

The most probable candidates for the WIMP are the neutralino, predicted by supersymmetric theories (SUSY) [35].

The direct method for WIMP search consists in detection for their elastic scattering on detector nuclei. The WIMP interaction probability in detector can be represented in a form:

$$
R=a_{p} W_{p}^{2}+a_{n} W_{n}^{2}+a_{0} V^{2}
$$

where the first two terms determine the spin-dependent (sd) scattering and the last one is the spin-independent (si) scattering [35]. The ratio of the number of spin-dependent WIMP scattering Rsd to the number of spin-independent scattering Rsi can be represent in a form:

$$
\operatorname{Rsd} / \mathrm{Rsi}=\eta_{\mathrm{A}} \cdot \eta_{\text {susY, }}
$$

where $\eta_{A}$ is determined by nuclear structure, and $\eta_{\text {SusY }}-$ by neutralino-quark interaction in SYSY model.

The dependence of $\eta_{A}$ from atomic number $A$ for nuclei $\left({ }^{1} \mathrm{H}, D_{2},{ }^{3} \mathrm{He},{ }^{73} \mathrm{Ge},{ }^{127} \mathrm{I}\right.$ and others) with nonzero spins is shown in Fig. 9 (Left) [35]. The dependence $R_{s d}(A) / R_{s d}\left(G e^{73}\right)$ on neutralino mass are shown in Fig. 9 (Right) for nuclei ${ }^{19} \mathrm{~F}$ and $\mathrm{Nal}$ [36]. One can see that more strong restrictions on spindependent part of WIMP interactions can be obtained in experiment with ${ }^{19} \mathrm{~F}\left(\mathrm{Ar}+\mathrm{CF}_{4}, \mathrm{Xe}+\mathrm{CF}_{4}\right)$ as 
compared with other nuclei $[20,37]$. The measurements are especially attractive in region of WIMP mass $8 \mathrm{GeV} \leq m_{x} \leq 14 \mathrm{GeV}$, where $R_{s d}>R_{s i}$.

At present time a great experiments [24, 26-28] are carried out for WIMP search with detector containing nuclei LAr, LXe, NaJ, Ge, the spin-independent (coherent) scattering for which is large $[28,29]$. One can see, that sensitivity of these experiments for spin-dependent scattering is 10-100 times less of expected effect as distinguished from coherent scattering experiments (see. Fig.9 right).The proposed in this work experiments increases the sensitivity of spin-dependent measurements to the point of the expected effect.

In this context, in our experiments $[21,25,39]$ as another filling of the chambers for search of low-mas WIMP $\left(<10 \mathrm{GeV} / \mathrm{c}^{2}\right)$ and solar axions with spin-dependent interaction with deuterium $\left(\mathrm{D}_{2}\right),{ }^{3} \mathrm{He}$, ${ }^{19} \mathrm{~F}$ and ${ }^{20} \mathrm{Ne}$ we propose to use the mixtures: ${ }^{20} \mathrm{Ne}+10 \% \mathrm{H}_{2}, \mathrm{D}_{2}+3 p p m T M A E,{ }^{3} \mathrm{He}+3 \% \mathrm{CH}_{4}$ at pressure 10-17 bar. And in our experiments [16-18] with liquid gases mixtures is used the mixtures with ${ }^{19} \mathrm{~F}: \mathrm{LAr}+$ $\mathrm{CF}_{4}$ and $\mathrm{LXe}+\mathrm{CF}_{4}$. The relative scintillation light outputs for investigated gases evaluated by an distribution area is shown in Fig 10.

In this section, we describe a double-phase xenon chamber with a system GEM +pin-anodes and system of photomultipliers. Fig. 11 shows a design of the chamber.
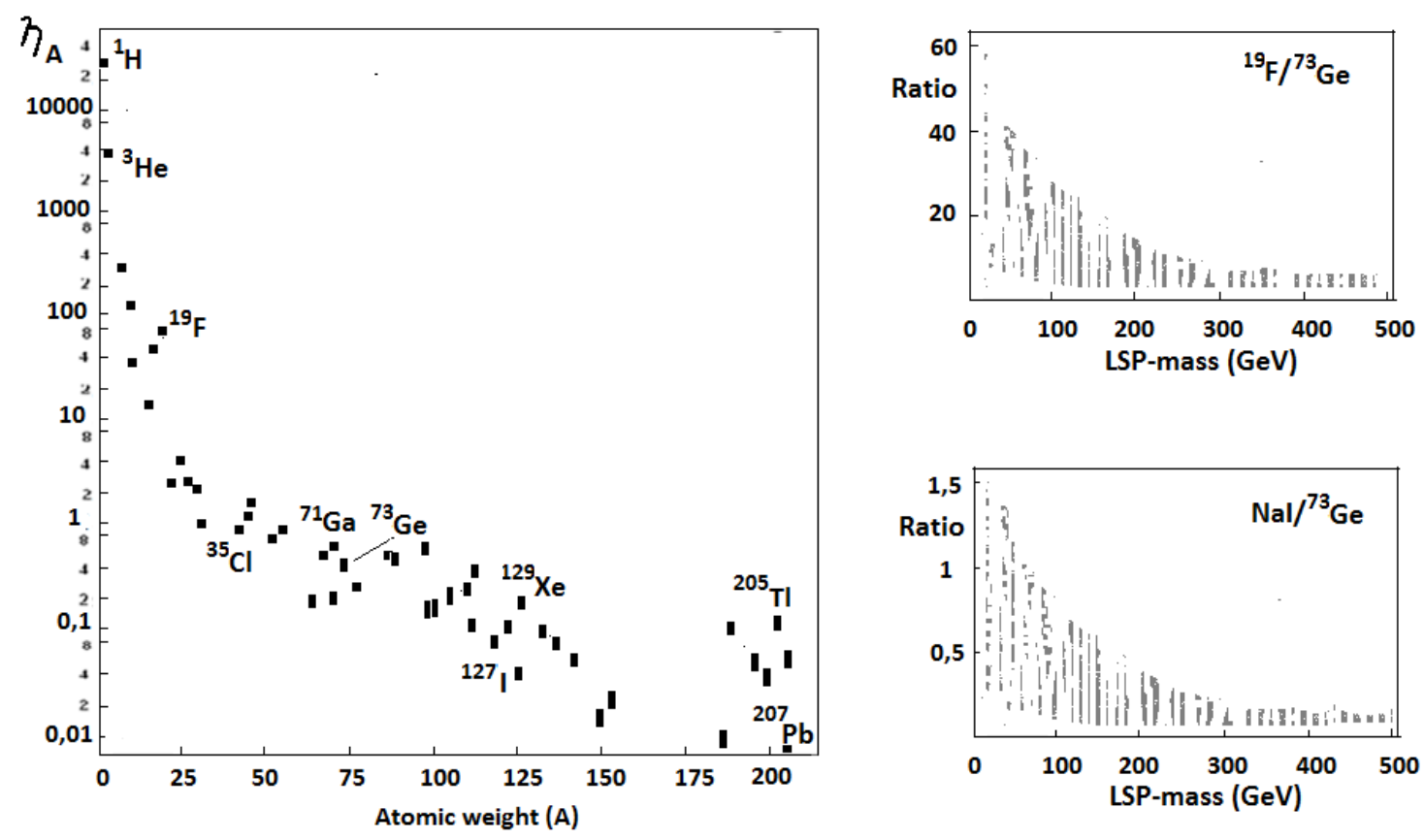

Fig. 9. Left: The dependence of nuclear factor $\eta_{A}$ from atomic number $A$ for the nuclei with nonzero spins [35]. The high of symbols presents the change of $\eta_{A}$ in a WIMP mass interval $10 \mathrm{GeV} \leq m_{x} \leq 500 \mathrm{GeV}$. Right: The dependence of Rsd (A)/ Rsd $\left({ }^{73} \mathrm{Ge}\right)$ from WIMP mass for ${ }^{19} \mathrm{~F}$ and $\mathrm{Nal}[36]$. 


\begin{tabular}{|l|c|c|c|c|c|c|}
\hline Gas & $\mathrm{Xe}$ gas & $\mathrm{LXe}$ & $\mathrm{Xe}+\mathrm{CF}_{4}$ & $\mathrm{LXe}+\mathrm{CF}_{4}$ & $\mathrm{LCF}_{4} / \mathrm{CF}_{4}$ & $\mathrm{LAr}+\mathrm{CF}_{4} / \mathrm{Ar}+\mathrm{CF}_{4}$ \\
\hline $\begin{array}{l}\text { Scintil. } \\
\text { output }\end{array}$ & 1 & 1 & 0,3 & 0,16 & 0,5 & 0,5 \\
\hline
\end{tabular}

Fig. 10. The ratio of relative scintillation light outputs for different gases mixtures [44].
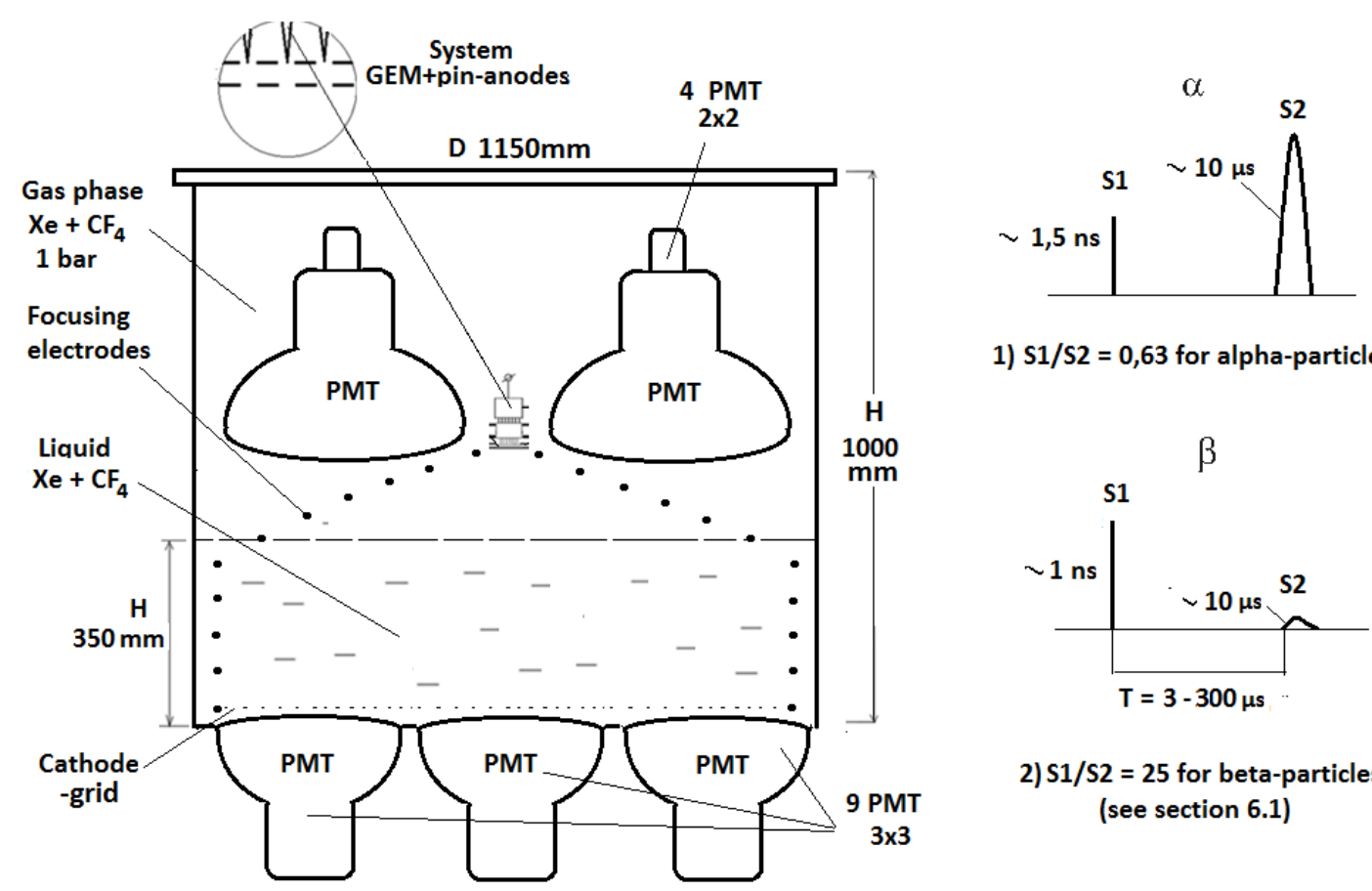

1) $S 1 / S 2=0,63$ for alpha-particles

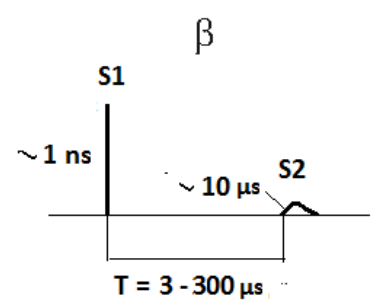

2) $\mathrm{S} 1 / \mathrm{S} 2=25$ for beta-particles (see section 6.1)

Fig. 11. Double-phase xenon chamber with a system GEM + pin-anodes and photomultipliers.

To suppress the $\beta$ background, we proposed a comparison of scintillation singlet signal (S1) and ionization signal (S2) for every event is suggested. The addition in $\mathrm{Xe}$ of $\mathrm{CF}_{4}$ and suppression of long triplet component of signals ( $27 \mathrm{~ns}$ ). For alpha particles, the ratio $A=S 1 / S 2$ was 0.63 and scintillation (S1) and $B=S 1 / S 2$ for beta particles 25 . The ratio beta to alpha was $B / A=25 / 0,63=40$ for $50 \% X e+50 \% C F_{4}$ gases mixture ( see section 6.1 and 6.2). The delay time between $\mathrm{S} 1$ and $\mathrm{S} 2$ is $T=3-300 \mu \mathrm{s}$, because: the drift time of electrons is $\mathrm{T}=0-300 \mu \mathrm{s}$ in $\mathrm{LXe}+\mathrm{CF}_{4}$, and the drift time electrons is $\mathrm{T}=1-3 \mu \mathrm{s}$ in gases phase $50 \% \mathrm{Xe}+50 \% \mathrm{CF}_{4}[44]$.

7.2 The double-phase chamber with $\mathrm{LCH}_{4}+$ TMAE mixture for search of spin-dependent WIMPnucleon interactions.

In work [46] it is shown that the absolute lower bound for the rate of direct DM detection is due to the spin-dependent WIMP-nucleon interaction, and a new-generation experiment aimed at detecting DM with sensitivity higher than $10^{-5}$ event/(kg - day) should have a nonzero-spin target to avoid missing of the DM signal. In work is claimed that for targets with spin- nonzero nuclei it might be the spindependent interaction that determines the lower bound for the direct detection rate when the cross section of the scalar interaction, which is usually assumed to be the dominant part, drops below 10 12-13 $\mathrm{pb}$ particular, from this work one can see that all fluorine-containing targets ( $\mathrm{LiF}, \mathrm{CF}_{4}, \mathrm{C}_{2} \mathrm{~F}_{6}$, and $\mathrm{CaF}_{2}$, etc.) have almost the same sensitivity to both the SD and SI WIMP-nucleus interactions. 
Among all materials considered a detector with a ${ }^{73} \mathrm{Ge},{ }^{129} \mathrm{Xe}$, or Nal target has better prospects to confirm or to reject the DAMA result [29] due to the largest values of the lower bounds for the total rate $\mathrm{R}(10,50)>0.06-0.08$ events/(kg day). If, for example, one ignores the SI WIMP interaction, then all materials have almost the same prospects to detect DM particles with the only exception of $\mathrm{CH}_{4}(\mathrm{see}$ Fig. 12. The results obtained are based on previous evaluations of the neutralino-proton (neutron) spin and scalar cross sections for the neutralino masses $\mathrm{m}_{\mathrm{x}}<200 \mathrm{GeV} / \mathrm{c}^{2}$.
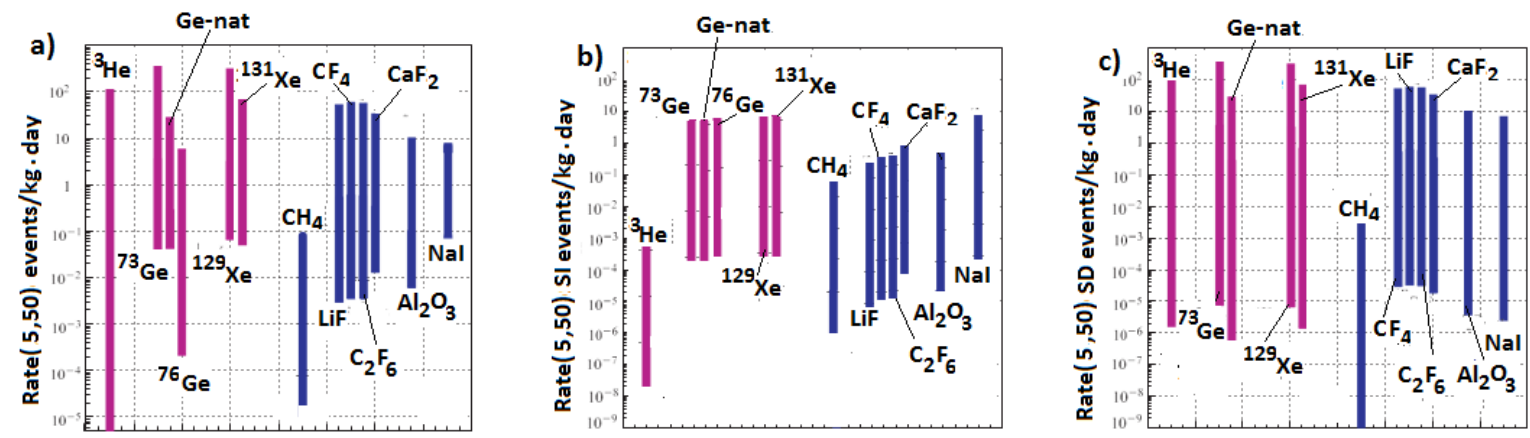

Fig. 12: a) variations of expected event rates, $R(5,50)$, for a number of targets followed from the DAMAallowed cross sections $\sigma_{\mathrm{SD}}$ and $\sigma_{\mathrm{SI}}$. Targets with nonzero-spin nuclei from the odd-neutron (odd-proton) group model are given in the left (right) part of the figure; b) variations of expected spin-independent contributions to the event rate, $\mathrm{R}(5,50) \mathrm{SI}$, in a number of targets followed from the DAMA-allowed cross sections $\sigma S D$ and $\sigma \mathrm{SI} ; \mathrm{c}$ ) the same as in b, but for the spin-dependent contributions $R(5,50) \mathrm{SD}$ [46].

In this context, in our experiment [15] with liquid $\mathrm{CH}_{4}$ as another filling of the chamber for search of low-mas WIMP $\left(<10 \mathrm{GeV} / \mathrm{c}^{2}\right)$ we propose to use the mixture $\mathrm{LCH}_{4}+40$ ppmTMAE (see Fig. 13).

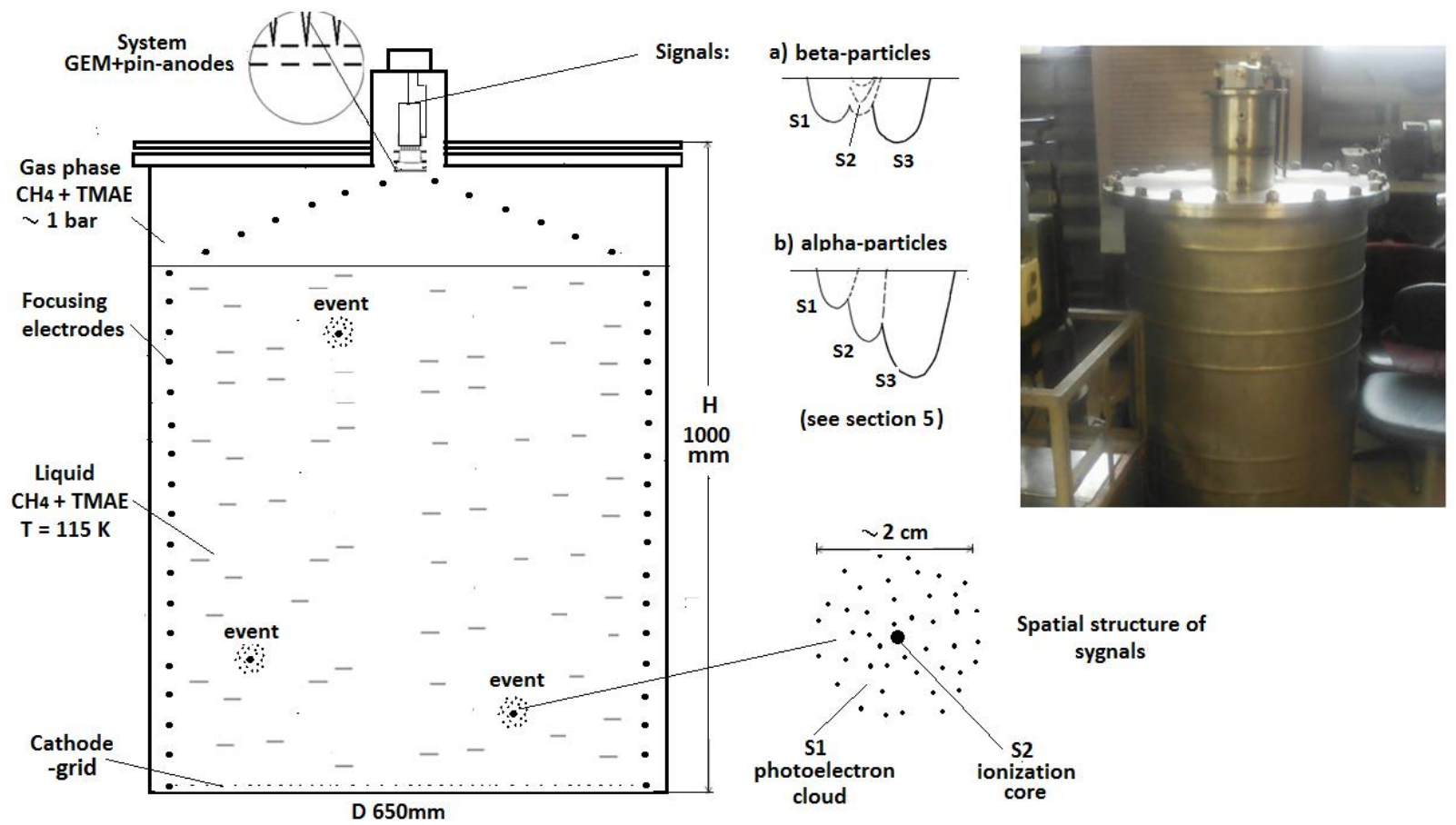

Fig. 13. A liquid-methane ionization chamber with a system GEM + pin-anodes for search of spindependent WIMP-nucleon interactions. 
The body of the chamber is made of titanium. The cathode-grid of the chamber is immersed in liquid methane. The layer of liquid methane above the cathode is equal to $800 \mathrm{~mm}$. The temperature of liquid methane is equal to $115 \mathrm{~K}$ and the pressure of gaseous methane over the liquid methane is equal to 1,3 bar for this temperature. The system GEM + pin-anodes is placed in gaseous methane. The addition in liquid $\mathrm{CH}_{4}$ of photosensitive TMAE [19] and suppression of triplet component of scintillation signals ensures the detection of scintillation signals with high efficiency and provides a complete suppression of the electron background (see section 5). As well as, in our experiments we use a system GEM + pin-anodes to more efficiently remove the background from the chamber electrodes and walls using positional veto of background signals.

\section{Time-projection chamber with high $\mathrm{dE} / \mathrm{dz}$ and energy resolutions.}

It is known that multi-wire proportional chambers (MWPC) used in time-projection chambers (TPC) fail to provide good energy resolution [34]. This is mainly due to the fact that multiplication of ionization electrons occurs in chamber regions with different electric field intensities resulting in variation of the multiplication factor. Additionally, the MWPC features a low $\mathrm{dEI} / \mathrm{dz}$ resolution in the drift direction of the ionization electrons due to slow motion of positive ion clouds from anode wires.

In our paper [38], we describe a TPC with both high energy and dE/dz resolutions. Fig. 14 shows a design of the chamber. The chamber contains the cathode 1 with a 4-mm-diameter collimating opening, behind which a 239Pu source with a $10^{5}$ flow rate is installed at a distance of $10 \mathrm{~mm}$. The rings 3 provide a uniform drift field. The MWPC 5 used for electron multiplication contains the cathode MWPC wound with a 2-mm pitch of a beryllium bronze $100-\mu \mathrm{m}$-diameter wire. The anode is winded of a $20 \mu \mathrm{m}$ diameter $\mathrm{W}+$ Au wire with a $2 \mathrm{~mm}$ pitch. The MWPC gap is $2 \mathrm{~mm}$. In projection, the anode wires are exactly in the middle between the cathode wires. The chamber contains cathode 6 and anode 7 of the measurement gap. The maximum electron multiplication factor for $\alpha$-particles is $\sim 10^{3}$. To get the total electron multiplication factor $\left(10^{5}\right)$, the chamber is set to MGEM 4 with the etching of holes [14].

Due to the photon mechanism, when the avalanche evolves in the anode MWPC wire, an electron charge proportional to the initial ionization charge appears in the transfer gap between the electrodes 5 and 6 . This charge drifts towards the measurement gap between the electrodes 6 and 7 . Electrodes 6 and 7 are winded of a $100 \mu \mathrm{m}$ diameter wire with a $1 \mathrm{~mm}$ pitch. The width of the gap 5-6 is $4 \mathrm{~mm}$. The electric field intensity in this gap is insufficient for evolving avalanches and the gap records the electrons without multiplication, i.e., in the induction operation mode of the ionization chamber. 


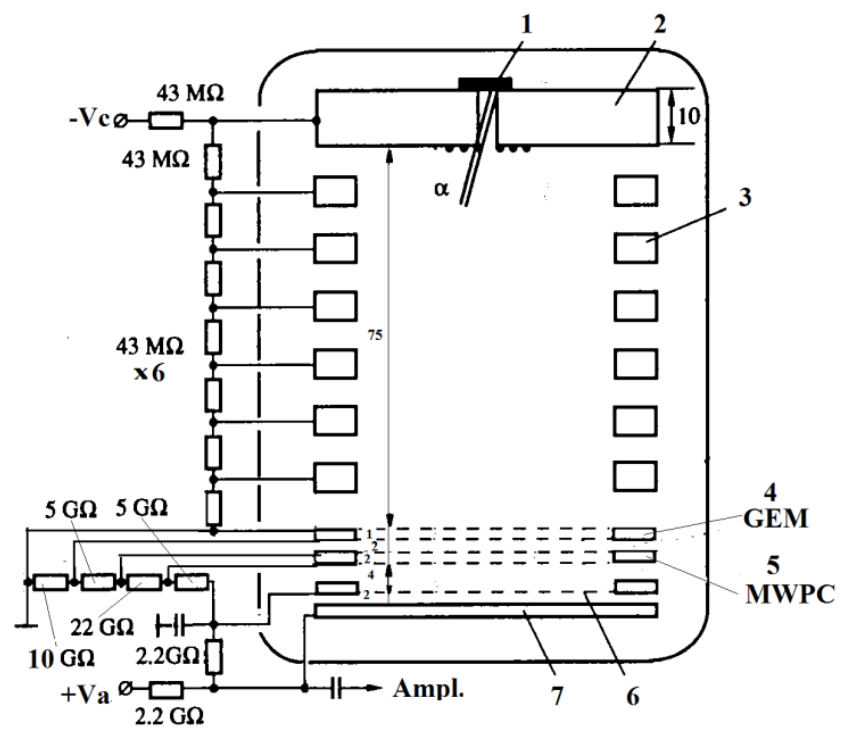

Fig. 14. Time projection chamber with MWPC and GEM. a) GEM

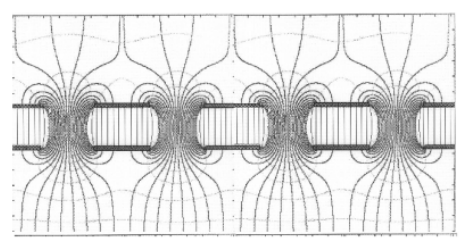

b) MWPC

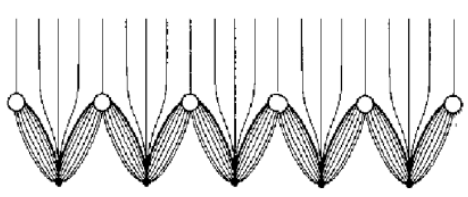

Fig. 151. Electric field structure in the GEM(a) and MWPC(b).

Fig. 15 shows the electric field structure in the MWPC and GEM gaps. Since the electric field intensity in the MWPC gap is much higher than in the drift gap and due to the symmetric location of the cathode relative to the anode wires, the electric field lines, when passing from the drift gap to the MWPC gap, concentrate into a narrow beam, which is orthogonal on the average to the anode plane and sufficiently uniform. As a result, the electron multiplication occurs in a more homogeneous electric field than in usual MWPCs, in which the electric field lines meet the anode wires at different angles in different MWPC regions and have different average densities.

In the process of its evolution, the avalanche discharge involves about $180^{\circ}$ of the anode wire in the azimuth. In our case, conditions for the evolving discharge are the same for all ionization electrons within the drift gap, because the field distributions on all wires are identical, and the fields are more homogeneous.

Since, for usually utilized mixtures, the electron drift speed in the measurement and drift gaps is $\sim \mathrm{n} \cdot 10^{6}$ and $\sim \mathrm{n} \cdot 10^{5} \mathrm{~cm} / \mathrm{s}$, respectively, and the measurement gap is small, the calculated $\mathrm{dE} / \mathrm{dz}$ resolution referred to the TPC drift gap appears to be sufficiently high: the chamber resolves the track regions or separate tracks spaced by $\sim 0.2 \mathrm{~mm}$.

In order to determine the energy resolution, the chamber was filled with an $\mathrm{Ar}+0.5 \% \mathrm{C}_{2} \mathrm{H}_{2}+$ $10 \% \mathrm{CH}_{4}$ mixture at a total pressure of 3,5 bar. When recording a-particles from a 239Pu source, the energy resolution was $8.1 \%$ (half-intensity half-width).

Three anode grids with $0,2 \mathrm{~mm}$ pitch and $0,2 \mathrm{~mm}$ gaps may be placed in the ionization chamber for detection of $x, y$ and $45^{\circ} x$-coordinates. The electrons are moving after multiplication through three anode grids inducting on them the signals. The errors for $x, y$-coordinates will be equal to $0,2 \mathrm{~mm}$ and error for $z$-coordinate will be $0,2 \mathrm{~mm}$ (not including diffusion errors).

The method allows you to measure the direction of movement of particles. In our work [45] we have study of TPC with the Penning mixture $\mathrm{He}+3 \% \mathrm{CH}_{4}$ filling at a pressure 17 bar for direct detection of solar neutrinos. As another filling is use the mixture ${ }^{3} \mathrm{He}+3 \% \mathrm{CH}_{4}$ for search of $\mathrm{SD}$-interactions. As well time projection chamber with the mixture $\mathrm{D}_{2}+3$ ppmTMAE filling at a pressure 10 bar allow to search of spin-dependent interactions of solar axions and deuterium $[21,23,25]$. 
Finally, we compare this chamber with existing multistage avalanche chambers, which also provide high energy and $\mathrm{dE} / \mathrm{dz}$ resolutions and with the MICROMEGAS chambers [7]. The MWPC used in our chamber in place of the avalanche chamber allows it to operate under an increased pressure of the filling gas (up to 10 - 20 bar), whereas other chambers are intended for operation mostly at pressures lower than the atmospheric pressure. The high resolution ( $d E / d x, d E / y, d E / z)$ of the TPC allows you to measure the direction of the flow of solar neutrinos and axions. Directionality allows to use only electrons recoiling away from the sun, effectively eliminating most background events.

\section{Detecting chambers for search of narrow pp-resonances (quarks) in the energy region 150 - $300 \mathrm{MeV}$.}

The existence of narrow peculiarities in a two-proton system was observed by JINR scientists in nucleon reactions with $\pi$-mesons production [40]. Over time the statistics has substantially been enlarged by this group and narrow pp-peaks at the level 3-5 standard errors have proved to be present in various reactions within a wide energy range [41]. Analyzing the effective mass distributions in the system of particles $n p, \pi^{-} \pi^{-}, \pi^{+} \pi^{-}$from inelastic reactions with $\pi$-mesons production this group also found the narrow peculiarities, excitation energies of which were just the same as those for pp-system. This group has observed narrow $\pi^{-}$p-resonances which excitation energies coincide well with those of pp-systems. In work [42] Y.A.Troyan group has observed the existence of narrow pp-resonances in elastic pp-scattering differential cross-section within 116-199 MeV.

The results are compared with the data of various experiments of elastic scattering at the energy region $0,2-10 \mathrm{GeV} / \mathrm{c}^{2}$ (Fig. 16).

The experiment with MPWC which detectors the recoil protons with mixture $\mathrm{Xe}+\mathrm{izo}-\mathrm{C}_{4} \mathrm{H}_{10}(1: 1)$ under a pressure 15 torr we proposed in year 1993 to search for narrow pp-resonances (quarks) in the energy region $150-300 \mathrm{MeV}$ at the MMF accelerator [43]. In the experiment the external proton beam of the accelerator is used with the energy changed from $160 \mathrm{MeV}$ to $300 \mathrm{MeV}$, intensity $2 \times 10^{11}$ protons per second and energy spread $0,5 \mathrm{MeV}$ (FWHM). The formed proton beam goes through the tube filled with hydrogen under a pressure of 5 bar (see Fig.13).

Recoil protons from the elastic pp-scattering are detected at angles $70^{\circ}$ laboratory system for coincidence with gas proportional and scintillation detectors. Detectors adsorb the recoil protons with the energies up to $35 \mathrm{MeV}$. This experiment requires high speed of ionization electrons collection time and respectively, the detector resolution time is about $10^{-8} \mathrm{sec}$. 

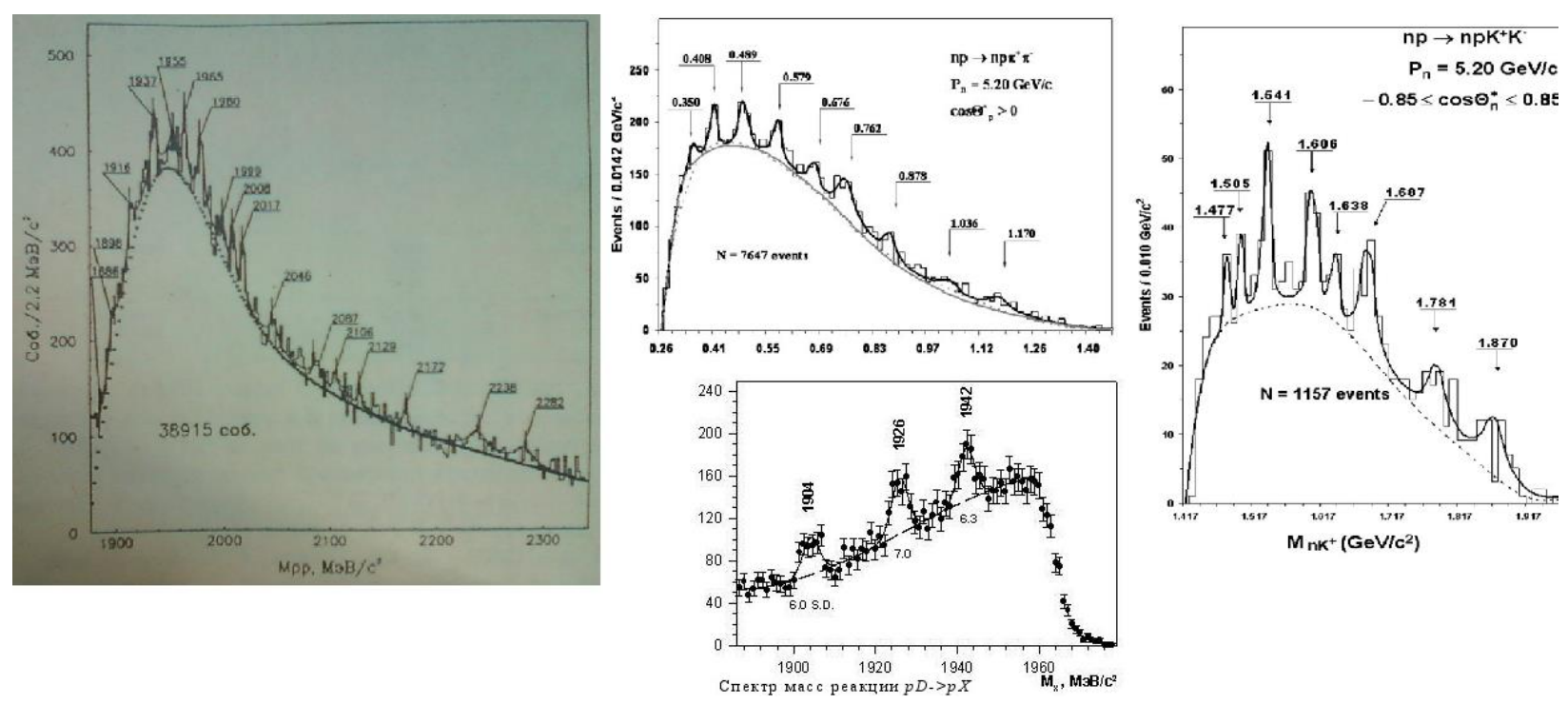

Fig. 16.The results of experiments JINR (Dubna) and MMF (INR, Moscow).

We propose detect the recoil protons with the energies up to $35 \mathrm{MeV}$ on scintillation chamber (see section 6.1) with $\mathrm{Xe}+\mathrm{CF}_{4}$ (1:1) filling under a pressure 50 torr and ionization chamber with system GEM+pin-anodes under a pressure 15 torr(see Fig.17). Signals are picked up from the scintillation and ionization chamber by fast amplifier and then are transferred to coincidence and anticoincidence circuits with a resolving time $10^{-8} \mathrm{sec}$. When the signal from the scintillation chamber coincides with that from the ionization chamber the signal amplitude of the latter is registered by the amplitude analyzer. Its readings determine the total number of the events at a certain energy and detection angle $\left(\mathrm{N}_{\text {events }}+\right.$ $\mathrm{N}_{\text {backgr }}$. The ratio $\mathrm{N}_{\text {events }} / \mathrm{N}_{\text {backgr }}$ is determined an elastic peak against the background.

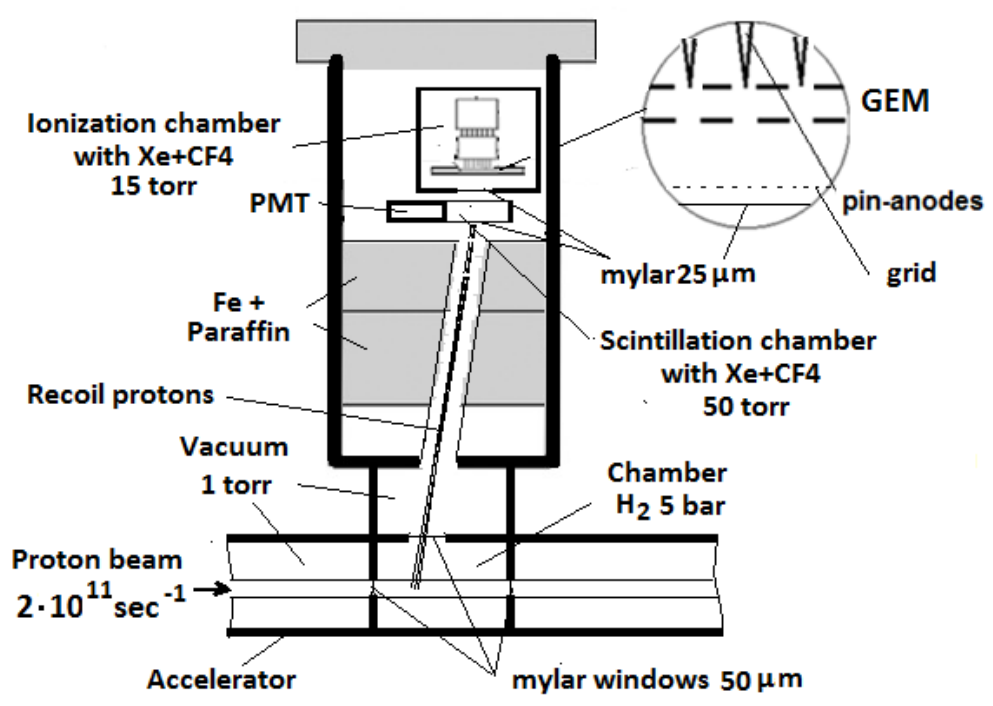

Fig.17. Experimental setup with scintillation and ionization chambers. 
We would like to mention that chambers with systems MGEM+pin-anodes, can have many applications for detecting events in accelerator experiments. As well as, the authors are currently studying the possibility of using spin-dependent reactions of light gases with nonzero-spin nuclei in high energy physic.

\section{Discussion}

In our works we have study the Penning mixtures: $\mathrm{He}+3 \% \mathrm{CH}_{4}, \mathrm{Ne}+30 \% \mathrm{H}_{2}, \mathrm{Ar}+10 \% \mathrm{Xe}, \mathrm{Ar}+$ $10 \% \mathrm{H}_{2}, 50 \% \mathrm{He}+50 \% \mathrm{Ne}[45]$ and $\operatorname{Ar}(\mathrm{Xe})+20 \% \mathrm{CF}_{4}, \operatorname{LAr}(\mathrm{LXe})+20 \% \mathrm{CF}_{4}[44]$.

In this work we propose as another filling of the chambers for search of low-mas WIMP $(<10$ $\left.\mathrm{GeV} / \mathrm{c}^{2}\right)$ and solar axions on spin-dependent interaction with deuterium $\left(D_{2}\right),{ }^{3} \mathrm{He},{ }^{19} \mathrm{~F}$ and ${ }^{21} \mathrm{Ne}$ is used the mixtures: ${ }^{21} \mathrm{Ne}+10 \% \mathrm{H}_{2}$ [21], $\mathrm{D}_{2}+3$ ppmTMAE [25], ${ }^{3} \mathrm{He}+3 \% \mathrm{CH}_{4}$ [39] at pressure 10-17 bar. And in our experiment with liquid mixtures $[16-18,37,44]$ is used the mixtures $\mathrm{LAr}+\mathrm{CF}_{4}\left({ }^{19} \mathrm{~F}\right)$ and $\mathrm{LXe}+\mathrm{CF}_{4}$ $\left({ }^{19} \mathrm{~F}\right)$.

The time projection chamber [38] with the mixture $D_{2}+3 p p m T M A E$ filling at a pressure 10 bar allow to search of spin-dependent interactions of solar axions and deuterium (section 8).

In work [46] is claimed that for targets with spin- nonzero nuclei it might be the spin-dependent interaction that determines the lower bound for the direct detection rate when the cross section of the scalar interaction, which is usually assumed to be the dominant part, drops below $10^{-12}-10^{-13} \mathrm{pb}$. In particular, from this work one can see that all fluorine-containing targets ( $\mathrm{LiF}, \mathrm{CF}_{4}, \mathrm{C}_{2} \mathrm{~F}_{6}$, and $\mathrm{CaF}_{2}$, etc.) have almost the same sensitivity to both the SD and SI WIMP-nucleus interactions. Among all materials considered a detector with a ${ }^{73} \mathrm{Ge},{ }^{129} \mathrm{Xe}$, or Nal target has better prospects to confirm or to reject the DAMA result [29] due to the largest values of the lower bounds for the total rate $R(10,50)>0.06-0.08$ events/(kg day). If, for example, one ignores the SI WIMP interaction, then all materials have almost the same prospects to detect DM particles with the only exception of $\mathrm{CH}_{4}$.

In this context, in our experiments [15] with liquid $\mathrm{CH}_{4}$ as another filling of the chamber for search of low-mas WIMP $\left(<10 \mathrm{GeV} / \mathrm{c}^{2}\right)$ we propose to use the mixtures $\mathrm{LCH}_{4}+40$ ppmTMAE (section 7.2).

The results obtained in our works [15-18] suggest that it is possible to develop large volume detectors capable of detecting scintillations with a $100 \%$ geometrical efficiency, by contrast to the well known detection techniques based on photomultipliers having efficiency of only a few percent. [27]

\section{Summary \& Outlook}

In our works [9-14] GEMs with wire (WGEM) or metal electrodes (MGEM) and gas gap between metal electrodes without plastic were realized. An absence of a plastic insulation between electrodes of these GEMs excludes leakage currents and spark breakdowns between the electrodes.

In our works $[16,17,21,25]$ it was suggested to search low mass WIMPs and solar axions with help of chambers with GEMs and systems WGEM (MGEM) + pin-anodes. In work [18] we proposed a addition in liquid $\mathrm{Xe}(\mathrm{Ar})$ of photosensitive dopants and a comparison of scintillation (S1) and ionization signals (S2) for every event is suggested. In our works $[15,25,37]$ it was suggested that the search for spin-dependent WIMP-nucleon interactions with help of detecting system GEM + pin-anodes can be performed. 
In that respect we would like to add the next important comments:

1. As far as WIMPs with large masses (> $10 \mathrm{GeV}$ ) experimentally were not found so far $[24,27$, $29,31]$, it is necessary to search the WIMP with small masses $\left(\leq 10 \mathrm{GeV} / \mathrm{c}^{2}\right)$.

2. The data of the new DAMA/LIBRA-phase2 confirm a peculiar annual modulation of the singlehit scintillation events in the (2-6) keV energy range (WIMP mass $<10 \mathrm{GeV} / \mathrm{c}^{2}$ ) satisfying all of the multiple requirements of the Dark Matter [29]. J.Va'vra have supposed [31] that this effect is explained by low mass WIMP $\left(\sim \mathrm{GeV} / \mathrm{c}^{2}\right)$ scattering on protons in $\mathrm{H}_{2} \mathrm{O}$ molecules $\left(\mathrm{H}^{+}\right)$.

3. As well as, it is necessary to search of WIMP with small masses $\left(\leq 10 \mathrm{GeV} / \mathrm{c}^{2}\right)$ in spindependent interactions between DM particles and gases with nonzero-spin nuclei $\left(\mathrm{H}_{2}, \mathrm{D}_{2},{ }^{3} \mathrm{He}, \mathrm{CF}_{4}, \mathrm{CH}_{4}\right.$, etc.) [46].

Finally, we would like to mention that MGEMs can have various applications in medicine. Such MGEMs can be used in different medical instruments for their use in X-ray surgery or Positron Emission Tomography (PET), where a high operation stability and reliability of the whole complex of instrument is required. Recently we have proposed a PET system, based on these MGEMs and BaF2-crystals [47].

\section{References.}

[1] G. Charpak and F. Sauli, Physics Letters 78B (1978) 523.

[2] R. Bouclier et al, "The Gas Electron Multiplier (GEM)", IEEE Trans. Nucl. Sci. NS-44, 646, 1997.

[3] F. Sauli, "GEM: A new concept for electron amplification in gas detectors", Nucl. Inst. Meth., A386, 531, 1997.

[4] F. Sauli, "The gas electron multiplier (GEM): Operating principles and Applications", Nucl. Instrum. Meth. A805, 2016.

[5]. L. Periale et al, Nucl. Instrum. Methods A 478, 377 (2002).

[6]. R. Chechik et al, Nucl. Instrum. Methods A 535, 303 (2004).

[7]. A. Breskin et al, Nucl. Instrum. Methods A 598, 107 (2009).

[8]. A. Di Mauro et al, Nucl. Instrum. Methods A 581, 225 (2007).

[9] B.M.Ovchinnikov, V.V.Parusov, "A Multichannel Wire Gas Electron Multiplier", Instruments and Experimental Techniques, Vol.53, №5 (2010), 653-656.

[10] B.M.Ovchinnikov, V.V. Parusov, "Multichannel gas electron amplifier" Patent of Russia RU 2417384, 11.03.2010.

[11] B.M.Ovchinnikov, V.V.Parusov," Multichannel Wire Gas Electron Multipliers with 1 and 3mm Gaps", Instruments and Experimental Techniques, Vol.53, №6 (2010), 836-839. 
[12] "B.M.Ovchinnikov, V.V.Parusov, "Multichannel Wire Gas Electron Multipliers", Instruments and Experimental Techniques, Vol.56, №6 (2013), 634-636.

[13] B.M.Ovchinnikov, V.V.Parusov,"Multichannel gas electron amplifiers with metal electrodes", Instruments and Experimental Techniques, Vol.54, № 1(2011),43-46.

[14] D.S.Kosolapov, B.M.Ovchinnikov, V.V.Parusov, V.I.Razin, "Multichannel gas electron amplifiers with metal electrodes", Instruments and Experimental Techniques, Vol.56, № 6(2013), 684-685.

[15] V.A.Bednyakov, B.M.Ovchinnikov, V.V.Parusov, "Methane ionization chamber to search for spin-dependent dark matter interactions", arXiv:hep-ph/0508052v1, 4 Aug 2005.

[16] B.M.Ovchinnikov, V.V. Parusov, "Methods for detecting events in double-phase argon chambers", Instruments and Experimental Techniques, Vol.56, № (2013), 516-520.

[17] B.M.Ovchinnikov, Yu. B. Ovchinnikov, V.V. Parusov,"Massive Liquid Ar and Xe Detectors for Direct Dark Matter Searches", JETP Letters, 2012, Vol.96, №. 3, pp. 149-152.

[18] B.M.Ovchinnikov, V.V.Parusov, "A method for background reduction in an experiment for WIMP search with a Xe(Ar) - liquid ionization chamber", Astroparticle Physics 10 (1999) 129.

[19] D.F.Anderson, "New photosensitive dopants for liquid argon", Nucl. Instrum. Methods Phys. Res. A245, 361 (1986).

[20] B.M.Ovchinnikov, V.V.Parusov, "A Study of Ar + C2H4 and Xe + CF4 Gas Mixtures", Instruments and Experimental Techniques, Vol.56, № 6(2013), 637-639.

[21] B.M.Ovchinnikov, I.I.Tkachev, V.V. Parusov," The Methods for Direct Detection of WIMP with Mass <0.5 GeV", Physics Journal, Vol. 1, No. 2, July 2015, pp. 31-34.

[22] I. Giomataris, I. Irastorza, I. Savvidis et al., "A novel large-volume Spherical Detector with Proportional Amplification read-out" // JINST 3:P09007, 2008

[23] V.A.Bednyakov, B.M.Ovchinnikov, V.V.Parusov,"Search for spin-dependent interaction of Dark Matter particles", Preprint INR №1144/2005.

[24] L.Baudis, "WIMP dark matter direct-detection searches in noble gases", Physics of the Dark Universe (2014) 4 50-59

[25] B.M.Ovchinnikov, V.V. Parusov," Search for Low Mass WIMP and Axions, Emitted from the Sun" Universal Journal of Physics and Application, Vol. 10(2), 2016, pp. 58 - 59.

[26] K. Abe, K. Hieda, K. Hiraide et al., "Search for solar axions in XMASS, a large liquid-xenon detector", Physics Letters B 721 (2013) 46-50.

[27] E. April et al., "The scintillation and ionization yield of liquid xenon for nuclear recoils" arXiv: 0807.0459v2 [astro-ph], 31 Dec 2008. (10.1016/j.nima.2008.12.197). 
[28] R. Bernabei, P. Belli, F. Cappella, V. Caracciolo, S. Castellano, R. Cerulli, C. J. Dai and A. d'Angelo, "Final model independent result of DAMA/LIBRA-phase1", Eur. Phys. J. C73 (2013) 12, 2648R.

[29] R. Bernabei et al. "Improved model-dependent corollary analyses after the first six annual cycles of DAMA/LIBRA-phase2", Nuclear Physics and Atomic Energy, 20(4), 317-348 - December 2019.

[30] R.K.Janev, W.D.Langer, K.Evans et al., "Elementary Processes in Hydrogen-Helium Plasmas", Springer Verlag, Berlin, Heidelberg, New York, London, Paris, Tokyo.

[31] J. Va'vra,"A New Possible Way to Explain the DAMA Results", Physics Letters B 735 (2014)181.

[32] F.Murtas, "Development of a gaseous detector based on Gas Electron Multiplier (GEM) Technology", LNF-INFN, 28 Nov 2002.

[33] Ovchinnikov B.M., Parusov V.V. "A device to measure the total content of electrically negative impurities in gases, that are not electrically negative". Instruments and Experimental Technique. 1995. T. 38. № 1. C. 183-189. Patent of Russia RUS 2258924 29.06.2004

[34] A.Bondar et al. "Light multi-GEM detector for high-resolution tracking systems" Nucl.Instrum.Meth.A556:495-497, 2006.

[35] V.A. Bednyakov, H.V. Klapdor-Kleingrothaus, S.G. Kovalenko, Phys. Lett. B329 (1994) 5.

[36] V.A. Bednyakov, H.V. Klapdor-Kleingrothaus, S.G. Kovalenko, Phys. Rev. 50, n 12 (1994) 7128.

[37] B.M. Ovchinnikov, V.V. Parusov, "Freon-filled detectors", Instruments and Experimental Techniques 39(1996):795-798.

[38] B.M.Ovchinnikov, V.V.Parusov, "Time-projection chamber with high $\mathrm{dE} / \mathrm{dz}$ and energy resolutions", Instruments and Experimental Techniques, Vol.43, № 1, 2000, pp. 24-25.

[39] B.M. Ovchinnikov, V.V. Parusov "Search of the WIMP and Ge ${ }^{76} 2 \beta$-decay with high pressure chamber. Prototype of solar neutrino detector" Preprint INR RAS 0944/1997.

[40] K.Beshliu et al., Preprint JINR . DI83-815, Dubna, 1983, K. Beshliu et al., "Channel cross section of np interaction reactions with $\mathrm{Pn}=1-5 \mathrm{GeV} / \mathrm{c}$," Sov. J. Nucl.Phys. 43, 565-568 (1986)

[41] Yu.A.Troyan et al. Proc. 10 Int. Seminar on High Energy, Dubna, 1990, p.149.

[42] Yu.A.Troyan et al., Yadernaya fisika, vol.54, issue 5 (1991) 1301.

[43] V.N. Aseev , S.V. Dvortsov , V.A. Krasnov, B.M. Ovchinnikov, P.N. Ostroumov, V.V. Parusov "Proposal of the experiment on search for narrow pp-resonances in differential cross-section of 
elastic pp-scattering at small angles within the energy region below meson-production threshold" Preprint INR RAS 814/1993.

[44] B.M.Ovchinnikov, V.V.Parusov, "The preparing of an experiment for search the spindependent interaction of WIMP”, Preprint INR №1097/2003.

[45] B.M. Ovchinnikov, V.V. Parusov, "Investigation of the proportional discharge mechanism in nonelectronegative gases" Nucl. Instrum. Methods A 485, 539 (2002).

[46] V.A. Bednyakov, "Spin in Dark Matter Problem", Physic of elementary particles and atomic nucleus, 2007, T.38. 3.

[47] B.M. Ovchinnikov, V.V. Parusov, "Design of PET-Detector with improved characteristics" Preprint INR RAS 1271/2016. 